\title{
A Modified Formula for Calculating Dielectric Properties of Granular Agricultural Products in the Microwave Band
}

\author{
Runeng ZHONG ${ }^{1}$, Qinhong ZHENG*2, Bin $\mathrm{YAO}^{2}$, Tai XIANG ${ }^{1}$ \\ ${ }^{1}$ School of energy and environmental science, Yunnan Normal University, Kunming, 650500, China. \\ ${ }^{2}$ Key Laboratory of Photoelectric Information Technology of Yunnan Province, Kunming, 650500, China.
}

\begin{abstract}
Dielectric properties are important characteristic parameters in the microwave-assisted application of hygroscopic agricultural media. To break through the limitation of traditional general effective medium (GEM) formula and develop the new calculation formula suitable for agricultural products, a modified formula, that is, modified general agricultural products effective medium (abbreviated as MGAPEM) formula, was presented in this study for the dielectric property calculation of granular agricultural products in the microwave band. The correctness, validity, and accuracy of MGAPEM formula were verified by using literature and experimental measurement data. Results show that the maximum errors of the dielectric constant and dielectric loss factor are $0.42 \%$ and $0.98 \%$, respectively, in the conditions of microwave frequency (2.0-12.2 Ghz) and moisture content $(2.0 \%-19.7 \%$, wet basis). The accuracy of MGAPEM is higher than some traditional theoretical formulas. The MGAPEM formula provides a theoretical formula for the dielectric property analysis of granular agricultural products in the microwave band at room temperature $\left(24^{\circ} \mathrm{C}\right)$.
\end{abstract}

\section{Introduction}

Dielectric properties are important parameters in microwave-assisted applications of hygroscopic agricultural media. In the past few decades, scholars have conducted numerous measures about the dielectric properties of agricultural products by different techniques, the agricultural materials are equated to a mixture of conductive particles surrounded by continuous inclusions (air) ${ }^{[1]}$, and some models and mixed formulas have proposed to calculate and predict the dielectric properties of granular agricultural products $^{[2-4]}$. The early models and theoretical studies of the dielectric properties of granular agricultural products mainly focused on the analysis of the linear relationship between the dielectric properties and density and the dielectric mixing equation of the two-component gassolid (air and particle) mixture ${ }^{[5]}$. Kent first proposed that the dielectric constant $\left(\varepsilon^{\prime}\right)$ and loss factor $\left(\varepsilon^{\prime \prime}\right)$ of fish meal are linearly related to density ${ }^{[6]}$. Klein proposed that the square root of dielectric properties is linear with density ${ }^{[7]}$. Nelson adopted the cubic root linear function [called $\left.\left(\varepsilon^{\prime}\right)^{1 / 3}-v s-\rho\right)$ ] to predict the dielectric constants of pulverized coal, whole wheat flour, and whole wheat, he proposed that the prediction accuracy of cubic root linear function is better than square root linear function ${ }^{[3]}$. In addition, Nelson used several different dielectric mixing equations and linear extrapolation functions to analyse the dielectric properties of granular agricultural products, and the results showed that the Landau-Lifshitz-Looyenga(LLL) equation is considered suitable for the analysis of the dielectric properties of grain-like particulate matter. However, the values of some parameters corresponding to different particulate matters are different in these formulas. These parameters must be determined through a large number of experimental measurements even for a specific particulate matter. In recent years, scholars have successively established polynomial expressions to determine the relationship between the dielectric properties and the frequency, moisture content, temperature, and density of various agricultural particles, such as grain, wheat, cotton seeds, and coffee, through experimental measurements ${ }^{[4]}$. However, the obtained expression is not general, and this expression is almost a formula for a class of particulate matter, and it cannot be used to predict the dielectric properties of other types of granular agricultural products; the prediction error of some expressions is relatively large $(3.0 \%-10.0 \%)^{[5]}$. To date, a large error between the predicted and the measured values can still be observed even if the famous Maxwell-Garnett, Bruggeman, Lichtenecker, and other mixed equations are used to predict the dielectric properties of agricultural products ${ }^{[5]}$.

During agricultural production and processing, reliance on experimental measurement methods alone to obtain the dielectric properties of agricultural products is unrealistic. Research on the prediction formula for the dielectric properties of granular agricultural products under different parameters has attracted the attention of scholars ${ }^{[4]}$. In the present work, a modified general

* Corresponding author: Qinhong ZHENG, Email: zhong_rn@126.com 
agricultural product effective medium (MGAPEM) mixing formula is used to analyse the dielectric properties of granular agricultural products in the microwave band. The effective dielectric properties of millet-air with different frequencies (2-6 Ghz), different moisture contents $(2.0 \%-19.7 \%$ wet basis $)$, and room temperature condition $\left(24{ }^{\circ} \mathrm{C}\right)$ are measured. The correctness, accuracy, and validity of the MGAPEM formula are verified by the results of several mixed formulas (functions).

\section{Materials and Methods}

\subsection{GEM theoretical Formula}

In 1990, McLachlan proposed the calculation of the efficiency conductance of particle-filled binary composites based on a comprehensive analysis of the relationship between the efficiency conductivity of binary composites with the conductivity, volume fraction, and spatial dimension of the components ${ }^{[9]}$. The general effective medium (GEM) theoretical formula for the composites is:

$$
f_{1} \frac{\varepsilon_{1}^{1 / t}-\varepsilon^{1 / t}}{\varepsilon_{1}^{1 / t}+A \varepsilon^{1 / t}}+\left(1-f_{1}\right) \frac{\varepsilon_{2}^{1 / t}-\varepsilon^{1 / t}}{\varepsilon_{2}^{1 / t}+A \varepsilon^{1 / t}}=0
$$

Where $A$ and $t$ are the parameters used to characterize the microstructure, particle distribution, and interconnectivity of each component of the mixture. $A=\left(1-f_{c}\right) / f_{c}$, Where $f_{c}$ is a parameter related to the critical volume fraction. McLachlan verified the correctness and validity of the theoretical formula through experimental methods. Zhao obtained the different dimensionless parameters (i.e., $A$ and $t$ ) corresponding to the different dielectric constant ratio $\left(\varepsilon_{i} / \varepsilon_{e}\right)$ composites using an Monte Carlo - Finite Element Method(MC-FEM) ${ }^{[10]}$. It can be see that the values of $A$ and $t$ must be obtained via numerous experimental measurements or numerical simulations for different composites.

\subsection{Proposition of MGAPEM Formula}

The GEM formula cannot be directly used to predict or calculate the efficient dielectric properties of particlefilled binary composites under arbitrary medium parameters (arbitrary $\varepsilon_{1}$ and $\varepsilon_{2}$ ). With regard to the limitation of the GEM formula, this study uses the functional expressions of particle material medium parameter $\varepsilon_{1}$ and matrix material medium parameter $\varepsilon_{2}$ to characterize unknown characteristic parameters $A$ and $t$. Moreover, given that the granular agricultural product naturally stacked in the air is a hygroscopic mixture, the moisture content is the main influencing factor of its dielectric properties, and the inclusion is air $\left(\varepsilon_{2}=1-j 0\right)$. Accordingly, the modified formula for calculating the effective media of agricultural products is as follows:

$$
\begin{gathered}
f_{1} \frac{\varepsilon_{1}^{\beta}-\varepsilon^{\beta}}{\varepsilon_{1}^{\beta}+A \varepsilon^{\beta}}+f_{2} \frac{1-\varepsilon^{\beta}}{1+A \varepsilon^{\beta}}=0 \\
A=2 \times\left(1+e^{1-1 / \varepsilon_{1}}\right), \quad \beta=0.45
\end{gathered}
$$

Where $\varepsilon$ is the efficient dielectric property of the mixture; $\varepsilon_{2}=1-j 0$ is the dielectric property of particulate matter; $f_{1}$ and $f_{2}$ are the volume fraction of the particulate matter and inclusion (air) in the mixture $\left(f_{1}+f_{2}=1\right)$, respectively; and $e$ is the natural constant.

\subsection{Materials and equipment}

Millet (Xinjiang Fenggu Agricultural Technology Co., Ltd, 1mm), Winter wheat ('Scout 66' \& 'Scoutland') and white rice, ('Lebonnet') were selected as the experimental objects. Using vector network analyzer (ZNB20, Rohde \& Schwarz Ltd, Germany), 85051B Coaxial Air Lines (Agilent Technology) and moisture meter (DHS-16, Penang, Malaysia Changzhou Electronic Instrument Co., Ltd, accuracy Range: 0 100) to measure the dielectric properties of agricultural materials. Using vernier calipers (MITUTOYO 500-173, Japan, accuracy of $0.02 \mathrm{~mm}$ ) to measure the particle specification parameters. The Matlab R2012a (Math Works, Massachusetts) and the Origin 8.5 (Amsterdam, Netherlands) were used to complete numerical calculation and data processing analysis.

\section{Results and Discussion}

\subsection{Literature Data Validation of the MGAPEM}

Three agricultural products reported by Nelson et al. were used for theoretical calculation and comparative analysis on the basis of the data provided in the literature: ground white rice ("Lebonnet," 12.2\%, 11.0 Ghz), whole kernel hard red winter wheat ("Scout 66," 11.5\%, 9.4 $\mathrm{Ghz}$ ) and ground hard red winter wheat ("Scountland," $10.9 \%, 11.7 \mathrm{Ghz})^{[8]}$. This undertaking was performed to verify the validity and accuracy of the MGAPEM formula in the analysis of the dielectric properties of granular agricultural products.

First, $\beta \in(0.4,0.6)$ were set in the MGAPEM formula on the basis of the efficient dielectric constant $\varepsilon^{\prime}$ data (i.e., 2.45, 2.59, 2.95, 3.18, 3.54, 3.97, and 4.34) of the Lebonnet under different volume fraction. The dielectric constants $\varepsilon_{1}^{\prime}$ of the Lebonnet particles were calculated using the MGAPEM formula. The comparative analysis shows that when $\beta=0.45$ and $A$ are equal to $5.6,5.7,5.9,6.0,6.1,6.2$, and 6.3 , the calculation result of the MGAPEM formula is consistent with the LLL equation and $\left(\varepsilon^{\prime}\right)^{1 / 3}-v s-\rho$ function. Therefore, the optimal parameters in the MGAPEM formula were determined as $A=2 \times\left(1+e^{1-1 / \varepsilon_{1}}\right)$ and $\beta=0.45$. 
Table 1 Dielectric properties of kernels estimated by extrapolation of linear density functions of the dielectric constant and loss factor and by calculation from indicated dielectric mixture equations from permittivity measurements on bulk samples of agricultural products at $24^{\circ} \mathrm{C}$

\begin{tabular}{|c|c|c|c|c|c|c|c|c|c|c|c|c|c|c|c|c|c|c|}
\hline \multirow{3}{*}{$\begin{array}{l}\text { Material of } \\
\text { agricultural } \\
\text { products }\end{array}$} & \multirow{3}{*}{\multicolumn{2}{|c|}{$\begin{array}{l}\text { Frequ Moist } \\
\text { ency ure } \\
\text { /Ghz (wet) }\end{array}$}} & \multirow{2}{*}{\multicolumn{4}{|c|}{$\begin{array}{l}\text { Extrapolation of linear } \\
\text { functions } \\
\left(\varepsilon^{\prime}\right)^{1 / 2}\left(\varepsilon^{\prime}\right)^{1 / 3}\left(\varepsilon^{\prime \prime}+g\right)^{1 / 2}\end{array}$}} & \multicolumn{10}{|c|}{ Dielectric mixture equations } & \multirow{2}{*}{\multicolumn{2}{|c|}{$\begin{array}{c}\begin{array}{c}\text { Deviating amplitude } \\
(\%)\end{array} \\
\text { MGAPEM VS LLL }\end{array}$}} \\
\hline & & & & & & & \multicolumn{2}{|c|}{ MGAPEM } & \multicolumn{2}{|c|}{ LLL } & \multicolumn{2}{|c|}{ Böttcher } & \multicolumn{2}{|c|}{ Bruggeman } & \multicolumn{2}{|c|}{ CRI } & & \\
\hline & & & $f_{1}$ & $\varepsilon_{1}^{\prime}$ & $\varepsilon_{1}^{\prime}$ & $\varepsilon_{1}^{\prime \prime}$ & $\varepsilon_{1}^{\prime}$ & $\varepsilon_{1}^{\prime \prime}$ & $\varepsilon_{1}^{\prime}$ & $\varepsilon_{1}^{\prime \prime}$ & $\varepsilon_{1}^{\prime}$ & $\varepsilon_{1}^{\prime \prime}$ & $\varepsilon_{1}^{\prime}$ & $\varepsilon_{1}^{\prime \prime}$ & $\varepsilon_{1}^{\prime}$ & $\varepsilon_{1}^{\prime \prime}$ & $\Delta \varepsilon_{1}^{\prime}$ & $\Delta \varepsilon_{1}^{\prime \prime}$ \\
\hline \multirow{7}{*}{$\begin{array}{l}\text { Whole-kernel } \\
\text { hard red } \\
\text { Winter wheat. } \\
\text { 'Scout 66' } \\
\text { (Nelson, } \\
\text { 1983b) }\end{array}$} & 9.4 & $11.5 \%$ & 0.51 & 4.81 & 4.98 & 0.85 & 4.759 & 0.669 & 4.752 & 0.672 & 4.713 & 0.654 & 5.227 & 0.896 & 4.439 & 0.581 & 0.15 & -0.45 \\
\hline & & & 0.54 & \pm 0.27 & \pm 0.13 & \pm 0.04 & 4.830 & 0.698 & 4.827 & 0.703 & 4.763 & 0.674 & 5.275 & 0.916 & 4.524 & 0.613 & 0.06 & -0.71 \\
\hline & & & 0.62 & & & & 4.902 & 0.742 & 4.906 & 0.749 & 4.799 & 0.703 & 5.252 & 0.916 & 4.649 & 0.669 & -0.08 & -0.93 \\
\hline & & & 0.66 & & & & 5.010 & 0.754 & 5.017 & 0.761 & 4.894 & 0.710 & 5.331 & 0.909 & 4.777 & 0.688 & -0.14 & -0.92 \\
\hline & & & 0.74 & & & & 4.969 & 0.774 & 4.977 & 0.781 & 4.856 & 0.731 & 5.187 & 0.882 & 4.798 & 0.724 & -0.16 & -0.90 \\
\hline & & & 0.82 & & & & 5.000 & 0.806 & 5.008 & 0.812 & 4.906 & 0.770 & 5.141 & 0.877 & 4.883 & 0.771 & -0.16 & -0.74 \\
\hline & & & 0.88 & & & & 5.053 & 0.865 & 5.060 & 0.869 & 4.982 & 0.836 & 5.144 & 0.912 & 4.975 & 0.840 & -0.14 & -0.46 \\
\hline \multirow{7}{*}{$\begin{array}{l}\text { Ground hard } \\
\text { red winter } \\
\text { wheat, } \\
\text { 'Scoutland' } \\
\text { (Nelson. } \\
\text { 1983b) }\end{array}$} & 11.7 & $10.9 \%$ & 0.42 & 4.49 & 4.64 & 0.71 & 4.516 & 0.559 & 4.497 & 0.559 & 4.549 & 0.575 & 5.037 & 0.791 & 4.165 & 0.472 & 0.42 & 0.00 \\
\hline & & & 0.53 & \pm 0.14 & \pm 0.09 & \pm 0.06 & 4.531 & 0.626 & 4.525 & 0.630 & 4.479 & 0.609 & 4.907 & 0.808 & 4.253 & 0.550 & 0.13 & -0.63 \\
\hline & & & 0.57 & & & & 4.607 & 0.615 & 4.605 & 0.619 & 4.535 & 0.591 & 4.959 & 0.775 & 4.346 & 0.547 & 0.04 & -0.65 \\
\hline & & & 0.63 & & & & 4.673 & 0.656 & 4.676 & 0.661 & 4.580 & 0.622 & 4.968 & 0.793 & 4.447 & 0.594 & -0.06 & -0.76 \\
\hline & & & 0.71 & & & & 4.608 & 0.720 & 4.613 & 0.726 & 4.512 & 0.682 & 4.810 & 0.825 & 4.439 & 0.669 & -0.11 & -0.83 \\
\hline & & & 0.79 & & & & 4.605 & 0.650 & 4.611 & 0.654 & 4.519 & 0.619 & 4.743 & 0.712 & 4.485 & 0.617 & -0.13 & -0.61 \\
\hline & & & 0.82 & & & & 4.644 & 0.666 & 4.650 & 0.671 & 4.563 & 0.638 & 4.761 & 0.720 & 4.539 & 0.638 & -0.13 & -0.75 \\
\hline \multirow{7}{*}{$\begin{array}{l}\text { Ground white } \\
\text { rice, } \\
\text { 'Lebonnet' } \\
\text { (Nelson, } \\
\text { 1988) }\end{array}$} & 11.0 & $12.2 \%$ & 0.47 & 4.78 & 4.97 & 0.94 & 5.017 & 0.908 & 5.005 & 0.912 & 5.004 & 0.905 & 5.620 & 1.300 & 4.632 & 0.773 & 0.24 & -0.44 \\
\hline & & & 0.51 & \pm 0.14 & \pm 0.12 & \pm 0.07 & 4.995 & 0.923 & 4.989 & 0.928 & 4.945 & 0.901 & 5.518 & 1.265 & 4.647 & 0.799 & 0.12 & -0.54 \\
\hline & & & 0.55 & & & & 5.015 & 0.930 & 5.013 & 0.937 & 4.937 & 0.894 & 5.480 & 1.229 & 4.697 & 0.817 & 0.04 & -0.75 \\
\hline & & & 0.61 & & & & 5.040 & 1.021 & 5.044 & 1.030 & 4.936 & 0.967 & 5.417 & 1.282 & 4.768 & 0.916 & -0.08 & -0.87 \\
\hline & & & 0.68 & & & & 4.998 & 0.989 & 5.005 & 0.999 & 4.883 & 0.932 & 5.279 & 1.175 & 4.783 & 0.908 & -0.14 & -1.00 \\
\hline & & & 0.77 & & & & 4.857 & 0.927 & 4.864 & 0.935 & 4.757 & 0.879 & 5.026 & 1.033 & 4.714 & 0.875 & -0.14 & -0.86 \\
\hline & & & 0.83 & & & & 4.994 & 0.942 & 5.002 & 0.949 & 4.904 & 0.901 & 5.122 & 1.019 & 4.884 & 0.904 & -0.16 & -0.74 \\
\hline
\end{tabular}

Second, the dielectric properties of the three agricultural particles were calculated using the MGAPEM formula, LLL equation, Böttcher equation , Bruggeman equation, Lichtenecker equation, and CRI formula. Table 1 shows the comparison of the calculated results of the different theoretical formulas (functions). The results show that the MGAPEM formula agrees with the calculated values of the $\varepsilon-v s-\rho$ function at the microwave frequencies and volume fractions of three agricultural products. In comparison with the LLL equation, the maximum error of the dielectric constant and dielectric loss factor obtained using the MGAPEM formula are $0.42 \%$ and $0.97 \%$, respectively. As shown in Table 1, the dielectric constant calculation results of MGAPEM and LLL for the three agricultural products are consistent with the $\left(\varepsilon^{\prime}\right)^{1 / 3}-v s-\rho$ function; whereas the dielectric loss factor calculation results of Böttcher for Scout 66 and Scountland are close to the function. Generally, the accuracy based on the deviation amplitude $(\%)$ of the calculated results from the different equations and the $\varepsilon-v s-\rho$ function is in the following order: MGAPEM, LLL, Böttcher, CRI, Bruggeman, and Lichtenecker. Relatively, CRI, Bruggeman, and Lichtenecker have large deviation amplitudes.

\subsection{Experimental Data Verification of MGAPEM}

The efficient dielectric properties of the millet-air mixture were experimentally measured under the different microwave frequencies $(2-6 \mathrm{Ghz})$, different moisture contents $(2.0 \%, 4.7 \%, 7.5 \%, 10.0 \%, 15.0 \%$, $19.7 \%$, wet basis), and room temperature condition $\left(24^{\circ} \mathrm{C}\right)$ using an un-calibrated coaxial transmission reflection method ${ }^{[11]}$. This undertaking was performed to further verify the accuracy of using the MGAPEM formula for analyzing the dielectric properties of agricultural particles under different frequencies and moisture contents. Table 2 shows the measurement results of the efficient dielectric constant of the milletair mixture, where $M$ is the moisture content, and $\rho$ is the density of mixture $\left(\mathrm{g} / \mathrm{cm}^{3}\right)$. Table 2 showed that the computer results of MGAPEM, LLL, $\left(\varepsilon^{\prime}\right)^{1 / 3}-v s-\rho$, and Böttcher in the moisture content $(2 \% \sim 19.7 \%)$ and the frequency (2-6 Ghz). In comparison with LLL equation, the maximum deviation amplitudes of MGAPEM for the dielectric constant and dielectric loss factor are $0.38 \%$ nd $0.98 \%$, respectively. Böttcher is follow, and the deviation amplitude of the calculated results of the remaining formulas is large. Generally, the MGAPEM formula can be used to analyze the dielectric properties of agricultural particles under different frequencies and moisture contents. However, the calculation results of MGAPEM show a slightly increasing trend as the moisture content increases, but the deviation range is less than $1.0 \%$.

\section{Conclusions}

This work proposes the MGAPEM formula for calculating and predicting the dielectric properties of granular agricultural products in the microwave band. We use the functional expressions of particle material medium parameter and matrix material medium parameter to characterize unknown characteristic parameters in GEM, and an ideal function expression and an optimal dimensionless parameter were obtained. The correctness, validity, and accuracy of MGAPEM formula were verified by using literature and experimental measurement data. The comparison of the calculated results of different formulas (functions) shows that the MGAPEM and LLL results are highly consistent under the conditions of microwave frequency and volume fraction calculation of all the studied subjects. The maximum errors of the calculated results for the dielectric constant and dielectric loss factor are $0.42 \%$ 
and $0.98 \%$, respectively. The MGAPEM formula provides a theoretical formula for the dielectric property analysis of granular agricultural products in the microwave frequency band at room temperature $\left(24^{\circ} \mathrm{C}\right)$.

However, the dielectric loss in agricultural products increases with the moisture content because the internal moisture molecules of hygroscopic substances highorder multiple moment contribution and multiple scattering effect additional loss under microwave, the accuracy of using the MGAPEM formula to predict the dielectric properties of agricultural product particles with high moisture content $(M>20 \%)$ needs to be further verified. Measurement techniques for the dielectric properties of single particles of agricultural products must also be developed.

Table 2 The comparison of dielectric properties of millet particles with different moisture content calculated by different dielectric mixing equations at $24^{\circ} \mathrm{C}$

\begin{tabular}{|c|c|c|c|c|c|c|c|c|c|c|c|c|c|c|c|}
\hline \multirow{3}{*}{$\begin{array}{l}\text { Moisture } \\
\text { (wet } \\
\text { basis) }\end{array}$} & \multirow{3}{*}{$\begin{array}{c}\text { Frequ } \\
\text { ency } \\
(\mathrm{GHz})\end{array}$} & \multirow{2}{*}{\multicolumn{2}{|c|}{$\begin{array}{l}\text { Measurement } \\
\text { values of } \\
\text { mixture }\end{array}$}} & \multicolumn{10}{|c|}{ The calculation values of dielectric properties of millet particles } & \multirow{2}{*}{\multicolumn{2}{|c|}{$\begin{array}{c}\text { Deviating } \\
\text { amplitude } \\
(\%)\end{array}$}} \\
\hline & & & & \multicolumn{2}{|c|}{ LLL } & \multicolumn{2}{|c|}{ Böttcher } & \multicolumn{2}{|c|}{ Bruggeman } & \multicolumn{2}{|c|}{ Lichtenecker } & \multicolumn{2}{|c|}{ MGAPEM } & & \\
\hline & & $\varepsilon^{\prime}$ & $\varepsilon^{\prime \prime}$ & $\varepsilon_{1}^{\prime}$ & $\varepsilon_{1}^{\prime \prime}$ & $\varepsilon_{1}^{\prime}$ & $\varepsilon_{1}^{\prime \prime}$ & $\varepsilon_{1}^{\prime}$ & $\varepsilon_{1} \varepsilon_{1}^{\prime \prime}$ & $\varepsilon_{1}^{\prime}$ & $\varepsilon_{1}^{\prime \prime}$ & $\varepsilon_{1}^{\prime}$ & $\varepsilon_{1}^{\prime \prime}$ & $\Delta \varepsilon_{1}^{\prime}$ & $\Delta \varepsilon_{1}^{\prime \prime}$ \\
\hline \multirow{5}{*}{$2.00 \%$} & 2.00 & 1.973 & 0.119 & 3.708 & 0.392 & 3.713 & 0.392 & 3.957 & 0.487 & 4.313 & 0.343 & 3.721 & 0.392 & 0.35 & 0.00 \\
\hline & 3.00 & 1.921 & 0.091 & 3.539 & 0.296 & 3.544 & 0.297 & 3.755 & 0.361 & 4.078 & 0.260 & 3.552 & 0.296 & 0.38 & 0.16 \\
\hline & 4.00 & 1.899 & 0.078 & 3.469 & 0.250 & 3.474 & 0.251 & 3.671 & 0.303 & 3.981 & 0.221 & 3.482 & 0.251 & 0.38 & 0.31 \\
\hline & 5.00 & 1.892 & 0.077 & 3.448 & 0.248 & 3.452 & 0.249 & 3.645 & 0.300 & 3.951 & 0.219 & 3.461 & 0.249 & 0.37 & 0.22 \\
\hline & 6.00 & 1.879 & 0.057 & 3.406 & 0.184 & 3.411 & 0.184 & 3.597 & 0.221 & 3.895 & 0.162 & 3.419 & 0.184 & 0.38 & 0.00 \\
\hline \multirow{5}{*}{$7.50 \%$} & 2.00 & 2.172 & 0.155 & 3.965 & 0.458 & 3.945 & 0.45 & 4.237 & 0.568 & 4.615 & 0.654 & 3.974 & 0.457 & 0.24 & -0.13 \\
\hline & 3.00 & 2.102 & 0.132 & 3.761 & 0.384 & 3.745 & 0.378 & 3.992 & 0.467 & 4.331 & 0.538 & 3.771 & 0.383 & 0.28 & -0.20 \\
\hline & 4.00 & 2.068 & 0.120 & 3.663 & 0.349 & 3.648 & 0.344 & 3.874 & 0.421 & 4.195 & 0.485 & 3.673 & 0.349 & 0.27 & -0.05 \\
\hline & 5.00 & 2.078 & 0.114 & 3.692 & 0.331 & 3.677 & 0.325 & 3.910 & 0.399 & 4.237 & 0.460 & 3.702 & 0.330 & 0.27 & -0.22 \\
\hline & 6.00 & 2.063 & 0.101 & 3.650 & 0.292 & 3.636 & 0.288 & 3.861 & 0.351 & 4.180 & 0.405 & 3.660 & 0.292 & 0.28 & -0.11 \\
\hline \multirow{5}{*}{$10.00 \%$} & 2.00 & 2.308 & 0.189 & 3.989 & 0.497 & 3.950 & 0.481 & 4.229 & 0.601 & 4.579 & 0.688 & 3.995 & 0.496 & 0.16 & -0.28 \\
\hline & 3.00 & 2.214 & 0.164 & 3.747 & 0.426 & 3.715 & 0.414 & 3.943 & 0.505 & 4.251 & 0.578 & 3.754 & 0.425 & 0.18 & -0.26 \\
\hline & 4.00 & 2.178 & 0.156 & 3.652 & 0.404 & 3.623 & 0.392 & 3.832 & 0.474 & 4.124 & 0.543 & 3.659 & 0.403 & 0.20 & -0.35 \\
\hline & 5.00 & 2.185 & 0.156 & 3.671 & 0.403 & 3.642 & 0.391 & 3.855 & 0.474 & 4.150 & 0.542 & 3.679 & 0.402 & 0.21 & -0.37 \\
\hline & 6.00 & 2.157 & 0.143 & 3.600 & 0.367 & 3.572 & 0.357 & 3.772 & 0.429 & 4.056 & 0.491 & 3.608 & 0.366 & 0.21 & -0.25 \\
\hline \multirow{5}{*}{$15.20 \%$} & 2.00 & 2.610 & 0.256 & 4.393 & 0.616 & 4.324 & 0.587 & 4.672 & 0.746 & 5.057 & 0.847 & 4.395 & 0.612 & 0.05 & -0.62 \\
\hline & 3.00 & 2.490 & 0.239 & 4.107 & 0.566 & 4.049 & 0.541 & 4.331 & 0.671 & 4.669 & 0.763 & 4.110 & 0.563 & 0.07 & -0.60 \\
\hline & 4.00 & 2.444 & 0.243 & 3.999 & 0.573 & 3.946 & 0.549 & 4.204 & 0.675 & 4.524 & 0.767 & 4.003 & 0.570 & 0.10 & -0.45 \\
\hline & 5.00 & 2.449 & 0.239 & 4.010 & 0.565 & 3.957 & 0.541 & 4.218 & 0.666 & 4.539 & 0.757 & 4.014 & 0.562 & 0.11 & -0.49 \\
\hline & 6.00 & 2.409 & 0.208 & 3.919 & 0.488 & 3.869 & 0.468 & 4.113 & 0.572 & 4.421 & 0.650 & 3.923 & 0.486 & 0.10 & -0.40 \\
\hline \multirow{5}{*}{$19.70 \%$} & 2.00 & 2.960 & 0.309 & 5.247 & 0.77 & 5.247 & 0.723 & 5.726 & 0.990 & 6.254 & 1.116 & 5.242 & 0.762 & -0.09 & -0.98 \\
\hline & 3.00 & 2.816 & 0.315 & 4.889 & 0.773 & 4.889 & 0.730 & 5.272 & 0.969 & 5.740 & 1.097 & 4.887 & 0.767 & -0.04 & -0.82 \\
\hline & 4.00 & 2.747 & 0.333 & 4.718 & 0.812 & 4.718 & 0.769 & 5.057 & 1.005 & 5.496 & 1.140 & 4.718 & 0.806 & 0.00 & -0.77 \\
\hline & 5.00 & 2.723 & 0.329 & 4.660 & 0.800 & 4.660 & 0.758 & 4.987 & 0.986 & 5.416 & 1.119 & 4.661 & 0.794 & 0.01 & -0.80 \\
\hline & 6.00 & 2.670 & 0.253 & 4.538 & 0.612 & 4.538 & 0.581 & 4.848 & 0.749 & 5.256 & 0.850 & 4.538 & 0.608 & 0.01 & -0.66 \\
\hline
\end{tabular}

\section{Acknowledgments}

Project supported by the National Natural Science Foundation of China (Nos.61961044, 31560305).

\section{References}

1. Trabelsi S., Nelson S. O. Microwave Dielectric Properties of Cereal Grains[J]. Transactions of the ASABE, 2012, 55(5):1989-1996.

2. GUO Wenchuan, ZHU Xinhua. Dielectric Properties of Red Pepper Powder Related to Radiofrequency and Microwave Drying[J]. Food \& Bioprocess Technology, 2014,7(12):3591-3601.

3. Nelson Stuart O. Dielectric properties of agricultural materials and their applications[M]. Elsevier: Academic Press, 2015.

4. Routray W., Orsat V. Recent advances in dielectric properties measurements and importance[J]. Current Opinion in Food Science, 2018,23:120-126.

5. Nelson Stuart O., Trabelsi Samir. Use of material dielectric properties in agricultural applications $[\mathrm{J}]$. Journal of Microwave Power and Electromagnetic Energy, 2016,50(4):237-268.

6. Kent, M. Complex Permittivity of Fish Meal: A General Discussion of Temperature Density and Moisture Dependence[J]. Journal of Microwave Power, 1977,12(4):341-345.
7. Klein K. Microwave Determination of Moisture in Coal: Comparison of Attenuation and Phase Measurement[J]. Journal of Microwave Power, 1981,16(3):289-304.

8. Nelson S. O. Correlating Dielectric Properties of Solids and Particulate Samples Through Mixture Relationships[J]. Transactions of the ASAE, 1992,35(2):625-629.

9. McLachlau DS, Blaskiewicz M., Newnham RE. Electrical Resistivity of Composites[J]. J. AM. Ceram.Soc., 1990,73(8):2187-2203.

10. Zhao Xuanhe, Wu Yugong, Fan Zhigang, et al. Three-dimensional simulations of the complex dielectric properties of random composites by finite element method[J]. Journal of Applied Physics, 2004,95(12):8110-8117.

11. Runeng Zhong, Qinhong Zheng, Bin Yao, et al. Measurement and analysis of dielectric property of granular agricultural products in the microwave frequency band[J]. Journal of Agricultural Science and Technology, 2019,21(12):68-75. 